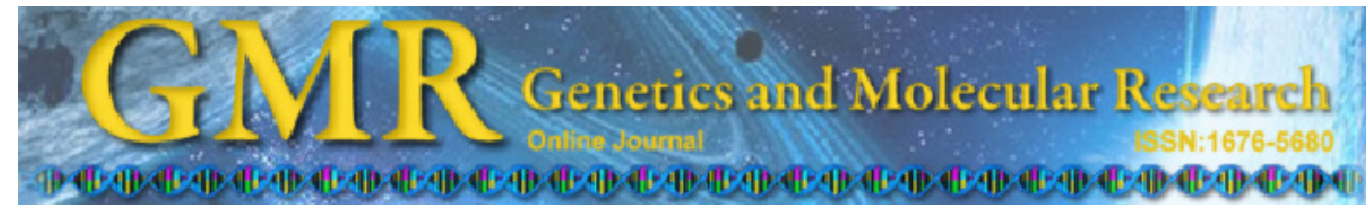

\title{
Vascular endothelial growth factor gene polymorphisms contribute to the risk of endometriosis: an updated systematic review and meta-analysis of 14 case-control studies
}

\author{
Y.Z. Li ${ }^{1}$, L.J. Wang ${ }^{2}$, X. Li ${ }^{3}$, S.L. Li ${ }^{1}$, J.L. Wang ${ }^{1}$, Z.H. Wu ${ }^{1}$, L. Gong ${ }^{1}$ and \\ X.D. Zhang ${ }^{1}$ \\ ${ }^{1}$ Department of Integrated Traditional Chinese and Western Medicine, \\ Qilu Hospital, Shandong University, Jinan, China \\ ${ }^{2}$ Department of Obstetrics and Gynecology, Qilu Hospital, \\ Shandong University, Jinan, China \\ ${ }^{3}$ Institute of Basic Medicine, Shandong Academy of Medical Science, \\ Jinan, China \\ Corresponding author: Y.Z. Li \\ E-mail: liyuzhu2003@126.com
}

Genet. Mol. Res. 12 (2): 1035-1044 (2013)

Received July 11, 2012

Accepted February 15, 2013

Published April 2, 2013

DOI http://dx.doi.org/10.4238/2013.April.2.20

\begin{abstract}
Endometriosis is a chronic gynecological disease defined as the presence of the endometrium outside the uterine cavity. Endometriosis is a multifactorial and polygenic disease in which angiogenesis may be implicated. Angiogenesis is under the control of numerous inducers, including vascular endothelial growth factor (VEGF). Many studies have reported that VEGF plays a role in the progression of the disease, but individually published studies showed inconclusive results. We investigated the association between VEGF polymorphisms and the susceptibility to endometriosis. The MEDLINE, EMBASE, Web of Science, and CBM databases were searched for all articles published up to June 25, 2012, which addressed VEGF polymorphisms and endometriosis risk. We investigated the
\end{abstract}


potential association between VEGF polymorphisms and the risk of endometriosis. Fourteen studies were included with a total of 3313 endometriosis cases and 3393 healthy controls. Meta-analysis results showed that the $\operatorname{rs} 699947(A>C)$ and $r s 1570360(\mathrm{G}>A)$ polymorphisms in the VEGF gene were associated with a decreased risk of endometriosis, while rs3025039 (C>T) might increase the risk of endometriosis. However, the rs833061 (T>C) and rs2010963 $(\mathrm{G}>\mathrm{C})$ polymorphisms of the VEGF gene did not appear to have an influence on endometriosis susceptibility. Results from the metaanalysis suggest that the rs3025039 $(\mathrm{C}>\mathrm{T})$ polymorphism of the VEGF gene increases the risk of endometriosis, but the rs699947 $(\mathrm{A}>\mathrm{C})$ and $\mathrm{rs} 1570360(\mathrm{G}>\mathrm{A})$ polymorphisms might be protective factors for endometriosis.

Key words: VEGF; Polymorphism; Endometriosis; Meta-analysis

\section{INTRODUCTION}

The endometrium is a complex tissue composed of different cells, including epithelial, stromal, inflammatory, perivascular, and blood vessel cells (Hsieh et al., 2004). Endometriosis is a chronic gynecological disease characterized by the growth of hormonally responsive, endometrial-like tissue outside the uterine cavity, causing diverse symptoms, including infertility, pelvic pain, and dysmenorrhea (Bhanoori et al., 2005). Histologically, endometriosis is a benign disease, but it can behave like a malignancy in terms of growing, infiltrating, and adhering to surrounding tissues (Varma et al., 2004). The prevalence of endometriosis ranges from 2 to $18 \%$ among women who seek tubal ligations, from 5 to $50 \%$ among infertile women, and from 5 to $20 \%$ among women with pelvic pain (Missmer and Cramer, 2003). The cause of endometriosis is likely multifactorial, involving environmental, immunological, endocrine, and genetic processes (Signorile and Baldi, 2010). The mechanisms underlying the disease remain unclear.

Vascular endothelial growth factor (VEGF), also known as vascular permeability factor, is a heparin-binding glycoprotein with potent angiogenic and endothelial cell-specific mitogenic activities that appears to play an important role in a variety of estrogen target tissues by locally regulating endometrial angiogenesis (Girling and Rogers, 2005; Kim et al., 2005). The VEGF gene is located on chromosome 6p21.3 and consists of 8 exons with alternate splicing, forming a family of proteins (Ferrara et al., 2003). At least 30 single nucleotide polymorphisms (SNPs) in this gene have been described (Watson et al., 2000). Several transcription factor-binding sites are found in the VEGF 5'-untranslated region (UTR), and variation within this region increases transcriptional activity (Fukumura et al., 1998). Polymorphisms within the 5'-UTR lead to differences in VEGF expression between individuals and may influence the etiology of a variety of pathological conditions with which VEGF has been associated (Bhanoori et al., 2005).

Numerous researchers have investigated the association between endometriosis and VEGF gene polymorphisms. However, the results remain controversial. Bhanoori et al. (2005) reported that patients with endometriosis had a higher frequency of the $+405 \mathrm{G}$ allele. Hsieh 
et al. (2004) reported that the $-460 \mathrm{~T}$ allele was associated with higher susceptibility to endometriosis. Furthermore, a positive association was found between the VEGF 936T allele and severe endometriosis in a Japanese population (Ikuhashi et al., 2007) but not in a Korean population (Kim et al., 2008). Therefore, in this study, we evaluated whether the VEGF gene polymorphism is a useful marker for predicting susceptibility to endometriosis.

\section{MATERIAL AND METHODS}

\section{Literature search strategy}

Relevant papers published before June 25, 2012 were identified by searching the MEDLINE, EMBASE, Web of Science, and CBM databases using the following expression: ("VEGF" OR "Vascular endothelial growth factor A" OR "Vascular permeability factor" OR "Vascular endothelial growth factor") AND ("Endometriosis" OR "Endometrioses" OR "Adenomyosis") AND ("Genetic polymorphism" OR "Single nucleotide polymorphism" OR "SNP" OR "Mutant" OR "Gene variation" OR "Gene mutation"). The referenced manuscripts in the identified articles or textbooks were also reviewed to find other potentially important studies.

\section{Inclusion and exclusion criteria}

The inclusion criteria were as follows: i) case-control study focused on associations between VEGF gene polymorphisms and endometriosis risk; ii) all patients with the diagnosis of endometriosis confirmed by pathological or histological examination; iii) the frequencies of alleles or genotypes in case and control groups could be extracted, and iv) published in English or Chinese. The exclusion criteria were as follows: i) no control population; ii) duplicate of previous publication; iii) based on incomplete data; iv) meta-analyses, letters, reviews, or editorial articles.

\section{Data extraction}

Using a standardized form, data from published studies were extracted independently by 2 reviewers to populate the necessary information. The following information was extracted from each of the articles included: 1st author, year of publication, country, language, ethnicity, study design, source of controls, number of cases and controls, mean age, sample, disease, genotype method, genotype frequency, and evidence of Hardy-Weinberg equilibrium (HWE) in the controls. In the case of conflicting evaluations, an agreement was reached following a discussion with a 3 rd reviewer.

\section{Quality assessment of included studies}

Two reviewers independently assessed the quality of the papers according to modified STROBE quality score systems (von Elm et al., 2007; Zhang et al., 2011). Forty assessment items related with the quality appraisal were used in this meta-analysis, scores ranging from 
0 to 40 . Scores of $0-20,20-30$, and 30-40 were defined as low, moderate, and high quality, respectively. Disagreement was resolved by discussion.

\section{Statistical analysis}

The meta-analysis examined the association between VEGF gene polymorphisms and the risk of endometriosis. The odds ratio (OR) and 95\% confidence interval $(95 \% \mathrm{CI})$ were calculated using Review Manager Version 5.1.6 (provided by the Cochrane Collaboration, available at: http://ims.cochrane.org/revman/download) and STATA Version 12.0 (Stata Corp, College Station, TX, USA). Inter-study variations and heterogeneities were estimated using the Cochran Q-statistic (Higgins and Thompson, 2002; Zintzaras and Ioannidis, 2005) ( $\mathrm{P}<0.05$ was considered a statistically significant heterogeneity). We also quantified the effect of heterogeneity using the $\mathrm{I}^{2}$ test, which ranges from 0 to $100 \%$ and represents the proportion of inter-study variability attributed to heterogeneity rather than to chance. A significant Q-test $(\mathrm{P}<0.10)$ or $\mathrm{I}^{2}>50 \%$ indicated heterogeneity among studies, and the randomeffects model was then conducted for meta-analysis; otherwise, the fixed-effects model was used. We tested whether genotype frequencies of the controls were in HWE using the $\chi^{2}$ test. Begg's funnel plots are often used to detect publication bias. However, due to the limitations of these plots, caused by varied sample sizes and subjective reviews, the Egger linear regression test, which measures funnel plot asymmetry using the natural logarithm scale of OR, was used to evaluate publication bias (Peters et al., 2006). When $\mathrm{P}<0.1$, publication bias was considered to be significant. All P values were 2 -sided. To ensure reliability and accuracy, 2 reviewers populated the data in the statistical software programs independently; these 2 reviewers obtained the same results.

\section{RESULTS}

\section{Studies included in the meta-analysis}

According to the inclusion criteria, 14 studies were included (Hsieh et al., 2004; Bhanoori et al., 2005; Kim et al., 2005; Ikuhashi et al., 2007; Gentilini et al., 2008; Kim et al., 2008; Zhao et al., 2008; Cosin et al., 2009; Kang et al., 2009; Liu et al., 2009a,b; Attar et al., 2010; Lamp et al., 2010; Altinkaya et al., 2011). The flow chart of study selection is shown in Figure 1. The total endometriosis cases and healthy controls were 3313 and 3393, respectively, in the 14 case-control studies that evaluated the relationship between VEGF polymorphisms and the risk of endometriosis. The publication year of the involved studies ranged from 2004 to 2011. All cases fulfilled the diagnosis criteria of endometriosis confirmed by pathological or histological examination. There were 5 SNPs in the VEGF gene in these 14 studies, including rs833061 $(\mathrm{T}>\mathrm{C})$ in 10 studies, rs2010963 $(\mathrm{G}>\mathrm{C})$ in 9 studies, rs3025039 $(\mathrm{C}>\mathrm{T})$ in 7 studies, rs699947 (A>C) in 4 studies, and rs $1570360(\mathrm{G}>\mathrm{A})$ in 3 studies. Eight of the 14 case-control studies were conducted with Asians and others were conducted with Caucasians. The HWE test was performed on the genotype distribution of the controls in all included studies, and all were in HWE $(\mathrm{P}>0.05)$. The characteristics and methodological quality of the included studies are summarized in Table 1. 


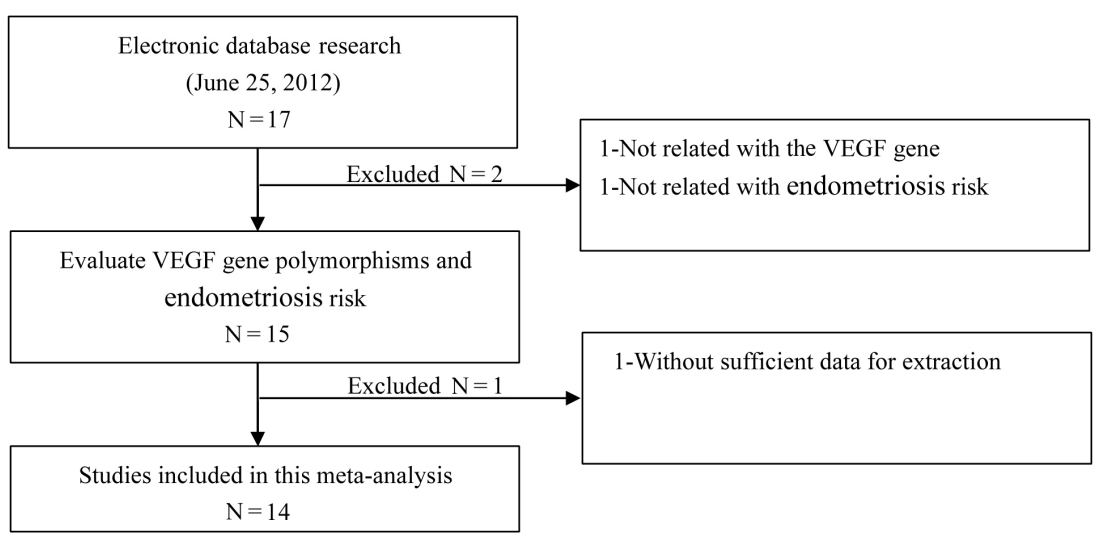

Figure 1. Flow chart shows study selection procedure.

\begin{tabular}{|c|c|c|c|c|c|c|c|c|c|}
\hline Reference & Country & Ethnicity & Case & Control & Source of control & Sample & Detection & SNP & $\begin{array}{l}\text { Quality } \\
\text { scores }\end{array}$ \\
\hline Hsieh et al., 2004 & China & Asian & 122 & 131 & Hospital-based & Blood & PCR-RFLP & rs833061 (T>C) & 23 \\
\hline Bhanoori et al., 2005 & India & Asian & 215 & 210 & Population-based & Blood & PCR-RFLP & $\begin{array}{l}\mathrm{rs} 833061(\mathrm{~T}>\mathrm{C}) \\
\mathrm{rs} 2010963(\mathrm{G}>\mathrm{C})\end{array}$ & 24 \\
\hline Kim et al., 2005 & Korea & Asian & 215 & 219 & Hospital-based & Blood & PCR-RFLP & $\begin{array}{l}\text { rs833061 }(\mathrm{T}>\mathrm{C}) \\
\text { rs2010963 }(\mathrm{G}>\mathrm{C})\end{array}$ & 22 \\
\hline Ikuhashi et al., 2007 & Japan & Asian & 147 & 181 & Population-based & Blood & PCR-RFLP & $\begin{array}{l}\text { rs833061 }(\mathrm{T}>\mathrm{C}) \\
\text { rs2010963 }(\mathrm{G}>\mathrm{C}) \\
\mathrm{rs} 3025039(\mathrm{C}>\mathrm{T})\end{array}$ & 26 \\
\hline Gentilini et al., 2008 & Italy & Caucasian & 203 & 140 & Hospital-based & Blood & PCR-RFLP & rs $2010963(\mathrm{G}>\mathrm{C})$ & 25 \\
\hline Kim et al., 2008 & Korea & Asian & 105 & 101 & Hospital-based & Blood & PCR-RFLP & $\mathrm{rs} 3025039(\mathrm{C}>\mathrm{T})$ & 28 \\
\hline Zhao et al., 2008 & Australia & Caucasian & 958 & 959 & Population-based & Blood & MassArray & $\begin{array}{l}\text { rs699947 }(\mathrm{A}>\mathrm{C}) \\
\text { rs833061 }(\mathrm{T}>\mathrm{C}) \\
\text { rs2010963 }(\mathrm{G}>\mathrm{C}) \\
\text { rs3025039 }(\mathrm{C}>\mathrm{T})\end{array}$ & 31 \\
\hline Cosín et al., 2009 & Spain & Caucasian & 186 & 180 & Hospital-based & Blood & PCR-RFLP & $\begin{array}{l}\text { rs833061 }(\mathrm{T}>\mathrm{C}) \\
\mathrm{rs} 2010963(\mathrm{G}>\mathrm{C}) \\
\mathrm{rs} 3025039(\mathrm{C}>\mathrm{T})\end{array}$ & 33 \\
\hline Kang et al., 2009 & China & Asian & 174 & 199 & Population-based & Blood & PCR-RFLP & $\begin{array}{l}\text { rs699947 (C>A) } \\
\text { rs1570360 }(\mathrm{G}>\mathrm{A}) \\
\text { rs833061 }(\mathrm{T}>\mathrm{C}) \\
\mathrm{rs} 3025039(\mathrm{C}>\mathrm{T})\end{array}$ & 32 \\
\hline Liu et al., 2009a & China & Asian & 344 & 360 & Population-based & Blood & PCR-RFLP & $\begin{array}{l}\text { rs699947 }(\mathrm{C}>\mathrm{A}) \\
\mathrm{rs} 3025039(\mathrm{C}>\mathrm{T})\end{array}$ & 30 \\
\hline Liu et al., 2009b & China & Asian & 344 & 360 & Population-based & Blood & PCR-RFLP & $\begin{array}{l}\mathrm{rs} 833061(\mathrm{~T}>\mathrm{C}) \\
\mathrm{rs} 1570360(\mathrm{G}>\mathrm{A})\end{array}$ & 32 \\
\hline Attar et al., 2010 & Turkey & Caucasian & 52 & 60 & Population-based & Blood & PCR-RFLP & $\begin{array}{l}\text { rs833061 }(\mathrm{T}>\mathrm{C}) \\
\mathrm{rs} 2010963(\mathrm{C}>\mathrm{G})\end{array}$ & 33 \\
\hline Lamp et al., 2010 & Estonia & Caucasian & 150 & 199 & Hospital-based & Blood & PCR-RFLP & $\begin{array}{l}\text { rs699947 }(\mathrm{A}>\mathrm{C}) \\
\text { rs1570360 }(\mathrm{G}>\mathrm{A}) \\
\text { rs2010963 }(\mathrm{G}>\mathrm{C}) \\
\text { rs3025039 }(\mathrm{C}>\mathrm{T})\end{array}$ & 31 \\
\hline Altinkaya et al., 2011 & Turkey & Caucasian & 98 & 94 & Hospital-based & Blood & PCR-RFLP & $\begin{array}{l}\text { rs833061 (T>C) } \\
\text { rs2010963 (G>C })\end{array}$ & 36 \\
\hline
\end{tabular}

$\mathrm{PCR}=$ polymerase chain reaction; $\mathrm{RFLP}=$ restriction fragment length polymorphism.

\section{Association between VEGF polymorphisms and endometriosis risk}

A summary of the meta-analysis findings of the association between VEGF gene 
polymorphisms and endometriosis risk is provided in Table 2 . No heterogeneity was found in the comparisons $(\mathrm{P}>0.05)$; thus, the fixed-effects model was used.

\begin{tabular}{|c|c|c|c|c|c|c|c|c|c|c|c|c|c|c|c|c|c|c|c|}
\hline \multirow[t]{2}{*}{ Reference } & \multirow[t]{2}{*}{ SNP } & \multicolumn{8}{|c|}{ Case } & \multicolumn{8}{|c|}{ Control } & \multicolumn{2}{|c|}{ HWE test } \\
\hline & & Total & 1 & 2 & $1 / 1$ & $1 / 2$ & $2 / 2$ & TA & $\overline{\mathrm{MF}}$ & Total & 1 & 2 & $1 / 1$ & $1 / 2$ & $2 / 2$ & TA & $\overline{\mathrm{MF}}$ & $\chi^{2}$ & $\mathrm{P}$ \\
\hline Hsieh et al., 2004 & rs833061 $(\mathrm{T}>\mathrm{C})$ & 122 & 190 & 54 & 68 & 54 & 0 & 244 & 0.22 & 131 & 179 & 83 & 48 & 83 & 0 & 262 & 0.32 & 28.166 & 0.000 \\
\hline \multirow[t]{2}{*}{ Bhanoori et al., 2005} & rs833061 $(\mathrm{T}>\mathrm{C})$ & 215 & 224 & 206 & 56 & 112 & 47 & 430 & 0.48 & 210 & 224 & 196 & 56 & 112 & 42 & 420 & 0.47 & 1.071 & 0.301 \\
\hline & $\mathrm{rs} 2010963(\mathrm{G}>\mathrm{C})$ & 215 & 351 & 79 & 140 & 71 & 4 & 430 & 0.18 & 210 & 305 & 115 & 113 & 79 & 18 & 420 & 0.27 & 0.613 & 0.434 \\
\hline \multirow[t]{2}{*}{ Kim et al., 2005} & rs833061 $(\mathrm{T}>\mathrm{C})$ & 215 & 309 & 121 & 113 & 83 & 19 & 430 & 0.28 & 219 & 323 & 115 & 120 & 83 & 16 & 438 & 0.26 & 0.099 & 0.753 \\
\hline & rs2010963 $(\mathrm{G}>\mathrm{C})$ & 215 & 241 & 189 & 76 & 89 & 50 & 430 & 0.44 & 219 & 264 & 174 & 74 & 116 & 29 & 438 & 0.40 & 2.463 & 0.117 \\
\hline \multirow[t]{3}{*}{ Ikuhashi et al., 2007} & rs833061 $(\mathrm{T}>\mathrm{C})$ & 147 & 211 & 83 & 72 & 67 & 8 & 294 & 0.28 & 181 & 244 & 118 & 80 & 84 & 17 & 362 & 0.33 & 0.570 & 0.450 \\
\hline & rs2010963 $(\mathrm{G}>\mathrm{C})$ & 146 & 172 & 120 & 48 & 76 & 22 & 292 & 0.41 & 181 & 206 & 156 & 56 & 94 & 31 & 362 & 0.43 & 0.627 & 0.428 \\
\hline & & 147 & 216 & 78 & 80 & 56 & 11 & 294 & 0.27 & 181 & 289 & 73 & 118 & 53 & 10 & 362 & 0.20 & 1.485 & 0.223 \\
\hline Gentilir & rs2010963 (G>C) & 203 & 244 & 162 & 69 & 106 & 28 & 406 & 0.40 & 140 & 193 & 87 & 67 & 59 & 14 & 280 & 0.31 & 0.036 & 0.849 \\
\hline Kim et & $\mathrm{rs} 3025039(\mathrm{C}>\mathrm{T})$ & 105 & 169 & 41 & 66 & 37 & 2 & 210 & 0.20 & 105 & 169 & 41 & 66 & 37 & 2 & 210 & 0.20 & 1.547 & 0.214 \\
\hline \multirow[t]{4}{*}{ Zhao et al., 2008} & rs699947 $(\mathrm{A}>\mathrm{C})$ & 958 & 973 & 943 & & - & 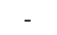 & 1916 & 0.49 & 959 & 951 & 967 & 236 & 479 & 244 & 1918 & 0.50 & 0.001 & 0.976 \\
\hline & rs 83306 & 953 & 956 & 950 & 227 & 502 & 224 & 1906 & 0.50 & 947 & 931 & 963 & 218 & 495 & 234 & 1894 & 0.51 & 1.978 & 0.160 \\
\hline & rs 2010 & 949 & 1306 & 592 & 442 & 422 & 85 & 1898 & 0.31 & 946 & 1331 & 561 & 459 & 413 & 74 & 1892 & 0.30 & 2.044 & 0.153 \\
\hline & $>\mathrm{T})$ & 957 & 1612 & 302 & 674 & 264 & 19 & 1914 & 0.16 & 945 & 1615 & 275 & 691 & 233 & 21 & 1890 & 0.15 & 0.068 & 0.795 \\
\hline \multirow[t]{3}{*}{ Cosín et al., 2009} & rs833061 $(\mathrm{T}>\mathrm{C})$ & 186 & 197 & 175 & 50 & 97 & 39 & 372 & 0.47 & 180 & 182 & 178 & 48 & 86 & 46 & 360 & 0.49 & 0.354 & 0.552 \\
\hline & C) & 186 & 245 & 127 & 77 & 91 & 18 & 372 & 0.34 & 180 & 248 & 112 & 84 & 80 & 16 & 360 & 0.31 & 0.245 & 0.621 \\
\hline & & 186 & 313 & 59 & 132 & 49 & 5 & 372 & 0.16 & 180 & 325 & 35 & 146 & 33 & 1 & 360 & 0.10 & 0.355 & 0.551 \\
\hline \multirow[t]{4}{*}{ Kang et al., 2009} & rs699947 (C>A) & 174 & 280 & 68 & 114 & 52 & 8 & 348 & 0.20 & 199 & 288 & 110 & 109 & 70 & 20 & 398 & 0.28 & 2.893 & 0.089 \\
\hline & & 174 & 297 & 51 & 128 & 41 & 5 & 348 & 0.15 & 199 & 302 & 96 & 117 & 68 & 14 & 398 & 0.24 & 0.880 & 0.348 \\
\hline & $\mathrm{rs}$ & 174 & 276 & 72 & 109 & 58 & 7 & 348 & 0.21 & 199 & 310 & 88 & 123 & 64 & 12 & 398 & 0.22 & 0.874 & 0.350 \\
\hline & & 174 & 276 & 72 & 109 & 58 & 7 & 348 & 0.21 & 199 & 336 & 62 & 140 & 56 & 3 & 398 & 0.16 & 0.972 & 0.324 \\
\hline \multirow{2}{*}{ Liu et al., 2009a } & rs699947 (C>A) & 344 & 556 & 132 & 223 & 110 & 11 & 688 & 0.19 & 360 & 531 & 189 & 200 & 131 & 29 & 720 & 0.26 & 1.304 & 0.254 \\
\hline & $r \times 3005039$ & 344 & 568 & 120 & 234 & 100 & 10 & 688 & 0.17 & 360 & 599 & 121 & 248 & 103 & 9 & 720 & 0.17 & 0.194 & 0.660 \\
\hline \multirow{2}{*}{ Liu et al., $2009 \mathrm{~b}$} & $\mathrm{rs} 833061(\mathrm{~T}>\mathrm{C})$ & 344 & 532 & 156 & 201 & 130 & 13 & 688 & 0.23 & 360 & 572 & 148 & 229 & 114 & 17 & 720 & 0.21 & 0.333 & 0.564 \\
\hline & & 344 & 577 & 111 & 239 & 99 & 6 & 688 & 0.16 & 360 & 560 & 160 & 221 & 118 & 21 & 720 & 0.22 & 0.965 & 0.326 \\
\hline \multirow[t]{2}{*}{ Attar et al., 2010} & $3061(\mathrm{~T}>\mathrm{C})$ & 52 & 68 & 36 & 27 & 14 & 11 & 104 & 0.35 & 60 & 71 & 49 & 30 & 11 & 19 & 120 & 0.41 & 23.107 & 0.000 \\
\hline & & 52 & 74 & 30 & 29 & 16 & 7 & 104 & 0.29 & 60 & 72 & 48 & 21 & 30 & 9 & 120 & 0.40 & 0.104 & 0.747 \\
\hline \multirow[t]{4}{*}{ Lamp et al., 2010} & rs699947 $(\mathrm{A}>\mathrm{C})$ & 150 & 188 & 112 & 56 & 76 & 18 & 300 & 0.37 & 199 & 210 & 188 & 61 & 88 & 50 & 398 & 0.47 & 2.535 & 0.111 \\
\hline & & 150 & 175 & 125 & 52 & 71 & 27 & 300 & 0.42 & 199 & 251 & 147 & 78 & 95 & 26 & 398 & 0.37 & 0.122 & 0.7 \\
\hline & & 150 & 241 & 59 & 94 & 53 & 3 & 300 & 0.20 & 199 & 293 & 105 & 108 & 77 & 14 & 398 & 0.26 & 0.003 & 0.9 \\
\hline & & 150 & 251 & 49 & 104 & 43 & 3 & 300 & 0.16 & 199 & 330 & 68 & 137 & 56 & 6 & 398 & 0.17 & 0.009 & 0.9 \\
\hline \multirow[t]{2}{*}{ Altinkaya et al., 2011} & rs833061 $(\mathrm{T}>\mathrm{C})$ & 98 & 190 & 6 & 92 & 6 & 0 & 196 & 0.03 & 94 & 186 & 2 & 92 & 2 & 0 & 188 & 0.01 & 0.011 & 0.917 \\
\hline & rs $2010963(\mathrm{G}>\mathrm{C})$ & 98 & 89 & 107 & 16 & 57 & 25 & 196 & 0.55 & 94 & 10 & 178 & 0 & 10 & 84 & 188 & 0.95 & 0.297 & 0.586 \\
\hline
\end{tabular}

$\mathrm{SNP}=$ single nucleotide polymorphism; HWE $=$ Hardy-Weinberg equilibrium; $1=$ wild allele; $2=$ mutant allele; $\mathrm{TA}=$ total alleles; $\mathrm{MF}=$ minor allele frequency.

The meta-analysis results showed that the $\mathrm{C}$ allele, the $\mathrm{C}$ allele carrier, and $\mathrm{CC}$ genotypes of the rs699947 (A>C) polymorphism of the VEGF gene were associated with decreased risk of endometriosis $(\mathrm{OR}=0.74,95 \% \mathrm{CI}=0.58-0.94, \mathrm{P}=0.01 ; \mathrm{OR}=0.68,95 \% \mathrm{CI}=0.55$ $0.84, \mathrm{P}<0.05 ; \mathrm{OR}=0.40,95 \% \mathrm{CI}=0.27-0.60, \mathrm{P}<0.05$, respectively). However, no association between rs699947 and endometriosis risk was found for the AC genotype (OR $=0.92$, $95 \% \mathrm{CI}=0.74-1.14, \mathrm{P}=0.43$ ). Moreover, the A allele carrier and the GA genotype of the rs $1570360(\mathrm{G}>\mathrm{A})$ polymorphism might also decrease the risk of endometriosis $(\mathrm{OR}=0.74$, $95 \% \mathrm{CI}=0.55-0.99, \mathrm{P}=0.04 ; \mathrm{OR}=0.81,95 \% \mathrm{CI}=0.67-0.97, \mathrm{P}=0.02$, respectively). We also found that the A allele and the AA genotype of rs 1570360 had no connection with the risk of endometriosis $(\mathrm{OR}=0.74,95 \% \mathrm{CI}=0.54-1.03, \mathrm{P}=0.07 ; \mathrm{OR}=0.49,95 \% \mathrm{CI}=0.19-1.24, \mathrm{P}$ $=0.13$, respectively). Interestingly, the results showed that there were positive associations among the $\mathrm{T}$ allele, the $\mathrm{T}$ allele carrier, and the $\mathrm{CT}$ genotype of the rs $3025039(\mathrm{C}>\mathrm{T})$ polymorphism with endometriosis risk $(\mathrm{OR}=1.16,95 \% \mathrm{CI}=1.04-1.30, \mathrm{P}=0.01 ; \mathrm{OR}=1.19,95 \% \mathrm{CI}$ $=1.05-1.36, \mathrm{P}<0.05 ; \mathrm{OR}=1.18,95 \% \mathrm{CI}=1.03-1.35, \mathrm{P}=0.02$, respectively). However, there 
was no evidence that the rs833061 $(\mathrm{T}>\mathrm{C})$ and $\mathrm{rs} 2010963(\mathrm{G}>\mathrm{C})$ polymorphisms of the VEGF gene were associated with risk of endometriosis $(\mathrm{P}>0.05)$.

Sensitivity analysis was performed by sequential omission of individual studies with various contrasts. However, the significance of the pooled OR in all individual analyses was not significantly influenced.

\section{Publication bias}

Publication bias was accessed based on the rs3025039, rs699947, and rs 1570360 polymorphisms in the VEGF gene by Begger's funnel plot and the Egger linear regression test. All funnel plots appeared symmetrical (Table 3). The Egger test also showed that there was no statistical evidence of publication bias (Table 4).

Table 3. Evaluation of publication bias by the Egger linear regression test.
\begin{tabular}{lccccc}
\hline SNP & Coefficient & SE & $t$ & $\mathrm{P}$ & $95 \% \mathrm{CI}$ \\
\hline rs3025039 (C>T) & & & & & \\
$\quad$ T allele & 1.151 & 1.248 & 0.920 & 0.399 & $-2.057,4.358$ \\
$\quad$ CT + TT & 0.906 & 1.125 & 0.810 & 0.457 & $-1.986,3.798$ \\
rs699947 (A>C) & & & & & $-1.046,-1.225$ \\
$\quad$ C allele & -4.135 & 0.676 & -6.110 & 0.026 & $-19.527,20.283$ \\
AC + CC & 0.378 & 1.567 & 0.240 & 0.849 & $-22.811,26.614$ \\
rs1570360 (G>A) & 1.902 & 5.743 & 0.330 & 0.772 & $-16.591,12.904$ \\
$\quad$ A allele & -1.843 & 3.428 & -0.540 & 0.645 & \\
GA + AA &
\end{tabular}

$\mathrm{SE}=$ standard error; $95 \% \mathrm{CI}=95 \%$ confidence interval.

Table 4. Meta-analysis of the association between VEGF gene polymorphisms and endometriosis risk.

\begin{tabular}{|c|c|c|c|c|c|}
\hline \multirow[t]{2}{*}{ Polymorphisms } & Case & Control & \multirow[t]{2}{*}{ OR $(95 \% \mathrm{CI})$} & \multirow[t]{2}{*}{$\mathrm{P}$} & \multirow[t]{2}{*}{ Effect model } \\
\hline & $\mathrm{n} / \mathrm{N}$ & $\mathrm{n} / \mathrm{N}$ & & & \\
\hline \multicolumn{6}{|l|}{ rs699947 (A>C) } \\
\hline $\mathrm{C}$ & $1255 / 3252$ & $1454 / 3434$ & $0.74(0.58,0.94)$ & 0.01 & \multirow{4}{*}{ Fixed } \\
\hline $\mathrm{AC}$ & $238 / 668$ & $289 / 758$ & $0.92(0.74,1.14)$ & 0.43 & \\
\hline $\mathrm{CC}$ & $37 / 668$ & $99 / 758$ & $0.40(0.27,0.60)$ & $<0.05$ & \\
\hline $\mathrm{AC}+\mathrm{CC}$ & $275 / 668$ & $388 / 758$ & $0.68(0.55,0.84)$ & $<0.05$ & \\
\hline \multicolumn{6}{|l|}{$\mathrm{rs} 833061(\mathrm{~T}>\mathrm{C})$} \\
\hline $\mathrm{C}$ & $2015 / 5700$ & $2088 / 5882$ & $0.97(0.90,1.06)$ & 0.53 & \multirow[t]{4}{*}{ Fixed } \\
\hline $\mathrm{TC}$ & $1253 / 2850$ & $1248 / 2941$ & $1.06(0.95,1.18)$ & 0.29 & \\
\hline $\mathrm{CC}$ & $381 / 2850$ & $420 / 2941$ & $0.90(0.77,1.05)$ & 0.16 & \\
\hline $\mathrm{TC}+\mathrm{CC}$ & $1634 / 2850$ & $1668 / 2941$ & $1.01(0.90,1.13)$ & 0.91 & \\
\hline \multicolumn{6}{|l|}{$\operatorname{rs} 1570360(\mathrm{G}>\mathrm{A})$} \\
\hline $\mathrm{A}$ & $398 / 2024$ & $563 / 2236$ & $0.74(0.54,1.03)$ & 0.07 & \multirow[t]{4}{*}{ Fixed } \\
\hline GA & $310 / 1012$ & $399 / 1118$ & $0.81(0.67,0.97)$ & 0.02 & \\
\hline $\mathrm{AA}$ & $44 / 1012$ & $82 / 1118$ & $0.49(0.19,1.24)$ & 0.13 & \\
\hline $\mathrm{GA}+\mathrm{AA}$ & $354 / 1012$ & $481 / 1118$ & $0.74(0.55,0.99)$ & 0.04 & \\
\hline \multicolumn{6}{|l|}{$\mathrm{rs} 2010963(\mathrm{G}>\mathrm{C})$} \\
\hline $\mathrm{C}$ & $1465 / 4428$ & $1536 / 4458$ & $0.75(0.54,1.04)$ & 0.09 & \multirow[t]{4}{*}{ Fixed } \\
\hline $\mathrm{GC}$ & $981 / 2214$ & $958 / 2229$ & $1.12(0.79,1.60)$ & 0.52 & \\
\hline $\mathrm{CC}$ & $242 / 2214$ & $289 / 2229$ & $0.60(0.29,1.23)$ & 0.16 & \\
\hline $\mathrm{GC}+\mathrm{CC}$ & $1223 / 2214$ & $1247 / 2229$ & $0.90(0.69,1.18)$ & 0.43 & \\
\hline \multicolumn{6}{|l|}{ rs3025039 $(\mathrm{C}>\mathrm{T})$} \\
\hline $\mathrm{T}$ & $721 / 4126$ & $675 / 4338$ & $1.16(1.04,1.30)$ & 0.01 & \multirow[t]{4}{*}{ Fixed } \\
\hline $\mathrm{CT}$ & $607 / 2063$ & $571 / 2169$ & $1.18(1.03,1.35)$ & 0.02 & \\
\hline TT & $57 / 2063$ & $52 / 2169$ & $1.19(0.81,1.74)$ & 0.37 & \\
\hline $\mathrm{CT}+\mathrm{TT}$ & $664 / 2063$ & $623 / 2169$ & $1.19(1.05,1.36)$ & $<0.05$ & \\
\hline
\end{tabular}

$\mathrm{OR}=$ odds ratio $; 95 \% \mathrm{CI}=95 \%$ confidence interval. 


\section{DISCUSSION}

Endometriosis (or adenomyosis) is a non-neoplastic condition characterized by the presence of ectopic endometrium in the myometrium with hyperplasia of the adjacent smooth muscle. In women, endometriosis can result in debilitating pelvic pain (both cyclical and noncyclical) and abnormal uterine bleeding and is paradoxically associated with both multiparity and infertility (Matalliotakis et al., 2005). Endometriosis, the most common tumor in women, has a prevalence of $10 \%$ in the general population and $30-40 \%$ in infertile women (Viganò et al., 2004). The establishment of new blood supply in the human exfoliated endometrium requires vascular proliferation and differentiation. Of known angiogenic factors, VEGF has emerged as important for the pathogenesis of endometriosis. VEGF is believed to play a central role in angiogenesis through a variety of mechanisms, including its effects on endothelial cell proliferation, survival, and migration (Ferrara, 2004).

Regulation of human VEGF is extremely complex and is a determinant of the rate and extent of angiogenesis, which is crucial for endothelial mitogenesis (Hsieh et al., 2004). VEGF is a potent angiogenic factor that promotes neovascularization, an important phenomenon involved in the implantation of endometrial cells in ectopic sites (Vigano et al., 2004). Polymorphisms in the 5'-UTR lead to differences in VEGF expression between individuals (Bhanoori et al., 2005). Recent studies have demonstrated a possible association between the VEGF gene polymorphism and endometriosis, where angiogenesis may be critical in disease development. Hsieh et al. (2004) demonstrated a significant association between the $-460 \mathrm{C} / \mathrm{T}$ polymorphism and susceptibility to endometriosis. Zhao et al. (2008) investigated the possible association between endometriosis and VEGF polymorphisms with a large Australian population sample and observed that these polymorphisms were not associated with the risk of endometriosis.

Given the controversial results in the published studies, we conducted a meta-analysis to explore the association between VEGF genetic polymorphisms and the risk of endometriosis. In this meta-analysis, which included a total of 3313 endometriosis cases and 3393 healthy controls and was based on 14 independent publications, we focused on the association of 5 polymorphisms in the VEGF gene with endometriosis risk. We demonstrated that the rs3025039 $(\mathrm{C}>\mathrm{T})$ polymorphism in the VEGF gene was associated with increased risk of endometriosis, while rs699947 (A>C) and $\mathrm{rs} 1570360(\mathrm{G}>\mathrm{A})$ might decrease the risk of endometriosis. However, the rs833061 ( $>C$ ) and rs2010963 (G>C) polymorphisms of the VEGF gene did not appear to influence endometriosis susceptibility. In interpreting the meta-analysis results, some limitations need to be noted. First, although the funnel plot and the Egger test did not show publication bias, selection bias could have occurred because only studies published in English or Chinese were included. Second, the number of published studies was not sufficient for analysis of specific cancer types. Third, our meta-analysis was based on unadjusted OR estimates because not all published articles contained adjusted ORs, or when they did, the ORs were not adjusted by the same potential confounders, such as pathological types, age, gender, geographic distribution. Finally, although all cases and controls are defined with similar inclusion criteria, potential factors that influence the results are not considered.

In summary, our meta-analysis of 14 case-control studies demonstrated that the rs3025039 (C>T) polymorphism of the VEGF gene might increase the risk of endometriosis, but rs699947 (A>C) and rs1570360 (G>A) might be protective factors. It is of importance to perform large sample studies to elucidate the influence of these polymorphisms on the susceptibility to endometriosis. 


\section{Conflicts of interest}

We declare that we have no conflicts of interest.

\section{ACKNOWLEDGMENTS}

Research supported by a grant from Shandong Medical Health Science and Technology Development Programs (\#2007HZ059).

\section{REFERENCES}

Altinkaya SO, Ugur M, Ceylaner G, Ozat M, et al. (2011). Vascular endothelial growth factor $+405 \mathrm{C} / \mathrm{G}$ polymorphism is highly associated with an increased risk of endometriosis in Turkish women. Arch. Gynecol. Obstet. 283: 267-272.

Attar R, Agachan B, Kuran SB, Toptas B, et al. (2010). Genetic variants of vascular endothelial growth factor and risk for the development of endometriosis. In Vivo 24: 297-301.

Bhanoori M, Arvind BK, Pavankumar Reddy NG, Lakshmi RK, et al. (2005). The vascular endothelial growth factor (VEGF) $+405 \mathrm{G}>\mathrm{C} 5$ '-untranslated region polymorphism and increased risk of endometriosis in South Indian women: a case control study. Hum. Reprod. 20: 1844-1849.

Cosin R, Gilabert-Estelles J, Ramon LA, Espana F, et al. (2009). Vascular endothelial growth factor polymorphisms $(-460 \mathrm{C} / \mathrm{T},+405 \mathrm{G} / \mathrm{C}$, and $936 \mathrm{C} / \mathrm{T})$ and endometriosis: their influence on vascular endothelial growth factor expression. Fertil. Steril. 92: 1214-1220.

Ferrara N (2004). Vascular endothelial growth factor: basic science and clinical progress. Endocr. Rev. 25: 581-611.

Ferrara N, Gerber HP and LeCouter J (2003). The biology of VEGF and its receptors. Nat. Med. 9: 669-676.

Fukumura D, Xavier R, Sugiura T, Chen Y, et al. (1998). Tumor induction of VEGF promoter activity in stromal cells. Cell 94: 715-725.

Gentilini D, Somigliana E, Vigano P, Vignali M, et al. (2008). The vascular endothelial growth factor $+405 \mathrm{G}>\mathrm{C}$ polymorphism in endometriosis. Hum. Reprod. 23: 211-215.

Girling JE and Rogers PA (2005). Recent advances in endometrial angiogenesis research. Angiogenesis 8: 89-99.

Higgins JP and Thompson SG (2002). Quantifying heterogeneity in a meta-analysis. Stat. Med. 21: 1539-1558.

Hsieh YY, Chang CC, Tsai FJ, Yeh LS, et al. (2004). T allele for VEGF gene-460 polymorphism at the 5'-untranslated region: association with a higher susceptibility to endometriosis. J. Reprod. Med. 49: 468-472.

Ikuhashi Y, Yoshida S, Kennedy S, Zondervan K, et al. (2007). Vascular endothelial growth factor $+936 \mathrm{C} / \mathrm{T}$ polymorphism is associated with an increased risk of endometriosis in a Japanese population. Acta Obstet. Gynecol. Scand. 86: $1352-1358$

Kang S, Zhao J, Liu Q, Zhou R, et al. (2009). Vascular endothelial growth factor gene polymorphisms are associated with the risk of developing adenomyosis. Environ. Mol. Mutagen. 50: 361-366.

Kim JG, Kim JY, Jee BC, Suh CS, et al. (2008). Association between endometriosis and polymorphisms in endostatin and vascular endothelial growth factor and their serum levels in Korean women. Fertil. Steril. 89: 243-245.

Kim SH, Choi YM, Choung SH, Jun JK, et al. (2005). Vascular endothelial growth factor gene +405 C/G polymorphism is associated with susceptibility to advanced stage endometriosis. Hum. Reprod. 20: 2904-2908.

Lamp M, Saare M, Laisk T, Karro H, et al. (2010). Genetic variations in vascular endothelial growth factor but not in angiotensin I-converting enzyme genes are associated with endometriosis in Estonian women. Eur. J. Obstet. Gynecol. Reprod. Biol. 153: 85-89.

Liu Q, Li Y, Zhao J, Sun DL, et al. (2009a). Association of polymorphisms -1154G/A and -2578C/A in the vascular endothelial growth factor gene with decreased risk of endometriosis in Chinese women. Hum. Reprod. 24: 2660-2666.

Liu Q, Li Y, Zhao J, Zhou RM, et al. (2009b). Association of single nucleotide polymorphisms in VEGF gene with the risk of endometriosis and adenomyosis. Zhonghua Yi Xue Yi Chuan Xue Za Zhi 26: 165-169.

Matalliotakis IM, Katsikis IK and Panidis DK (2005). Adenomyosis: what is the impact on fertility? Curr. Opin. Obstet. Gynecol. 17: 261-264.

Missmer SA and Cramer DW (2003). The epidemiology of endometriosis. Obstet. Gynecol. Clin. North Am. 30: 1-19, vii.

Peters JL, Sutton AJ, Jones DR, Abrams KR, et al. (2006). Comparison of two methods to detect publication bias in metaanalysis. JAMA 295: 676-680.

Signorile PG and Baldi A (2010). Endometriosis: new concepts in the pathogenesis. Int. J. Biochem. Cell Biol. 42: 778-780. 
Varma R, Rollason T, Gupta JK and Maher ER (2004). Endometriosis and the neoplastic process. Reproduction 127: 293-304.

Vigano P, Parazzini F, Somigliana E and Vercellini P (2004). Endometriosis: epidemiology and aetiological factors. Best. Pract. Res. Clin. Obstet. Gynaecol. 18: 177-200.

von Elm E, Altman DG, Egger M, Pocock SJ, et al. (2007). The Strengthening the Reporting of Observational Studies in Epidemiology (STROBE) statement: guidelines for reporting observational studies. Lancet 370: 1453-1457.

Watson CJ, Webb NJ, Bottomley MJ and Brenchley PE (2000). Identification of polymorphisms within the vascular endothelial growth factor (VEGF) gene: correlation with variation in VEGF protein production. Cytokine 12: 12321235 .

Zhang L, Liu JL, Zhang YJ and Wang H (2011). Association between HLA-B*27 polymorphisms and ankylosing spondylitis in Han populations: a meta-analysis. Clin. Exp. Rheumatol 29: 285-292.

Zhao ZZ, Nyholt DR, Thomas S, Treloar SA, et al. (2008). Polymorphisms in the vascular endothelial growth factor gene and the risk of familial endometriosis. Mol. Hum. Reprod. 14: 531-538.

Zintzaras E and Ioannidis JP (2005). Heterogeneity testing in meta-analysis of genome searches. Genet. Epidemiol. 28: $123-137$. 